\title{
Blind Spectrum Detector for Cognitive Radio Using Compressed Sensing and Symmetry Property of the Second Order Cyclic Autocorrelation
}

\author{
Ziad Khalaf, Amor Nafkha and Jacques Palicot \\ SUPELEC/IETR \\ SUPELEC, Avenue de la Boulaie, CS 47601 \\ 35576 Cesson Sévigné Cedex, France \\ Email: \{ziad.khalaf,amor.nafkha,jacques.palicot\}@ supelec.fr
}

\begin{abstract}
Based on the use of compressed sensing applied to recover the sparse cyclic autocorrelation (CA) in the cyclic frequencies domain on the one hand, and by exploiting the symmetry property of the cyclic autocorrelation on the other hand, this paper proposes a new totally blind narrow band spectrum sensing algorithm with relatively low complexity in order to detect free bands in the radio spectrum. This new sensing method uses only few iterations of the Orthogonal Matching Pursuit algorithm and have the particularity to perform robust detection with only few samples (short observation time). This new method outperforms the totally blind method proposed in [1] that only exploited the sparse property of the CA without requiring any additional calculation complexity for the same SNR and data samples number.
\end{abstract}

Index Terms-Cognitive Radio, Spectrum Sensing, Compressed Sensing, Sparsity, Detection Features, Orthogonal Matching Pursuit.

\section{INTRODUCTION}

Due to the rapid growth of wireless communications, spectrum resources are more and more needed. This indispensable necessity, in our days, has led to a scarcity in the spectrum resources. The objective of cognitive radio terminals is to get frequency bands in order to access the network in an autonomous and dynamic way. Most parts of the spectrum are allocated to licensed radio users (referred to as Primary Users (PUs)) that have exclusive access rights. However, Secondary Users (SUs) can still access opportunistically to the spectrum held by the PUs using spectrum sensing. There are many spectrum sensing techniques that enhance the SUs detection opportunities in to licensed bands, allowing the access to unused portions of the licensed spectrum [2], such as energy detection (ED) [3], [4], matched filtering (MF) detection [3], and cyclostationary detection (CSD) [5], [6]. Cyclostationary detection, requires high computation time and sufficient signal information and, it is not robust when the sample size is small [7]. One of the advantages of detecting using cyclostationarity is the possibility to discriminate users using the same modulation parameters. One solution is to integrate a specific signature in the physical layer. For example: in cognitive OFDM systems it is possible to embed signatures onto pilot tones, these signatures are easily intercepted implying short detection latency. This kind of technique avoids adding any side information dedicated for detection that would reduce the system capacity. In [8] two complementary signature/detection schemes are proposed based on second and third order statistics, the first scheme relies on redundancy between pilot symbols and the second is based on the use of maximumlength sequences.

As for the others well known detection methods cited above, we have the MF, which is an optimal detection method but needs an exact knowledge of the transmitted signal and requires synchronization which is difficult to achieve. Furthermore, in most cases, SUs can't know many information about the PUs signal structure. In the end, the ED is the simplest detection method but needs a perfect knowledge of the noise level. In fact, a small error in the estimation of the noise level is known to seriously impact the detection performance [4].

Recently, in our previous work [1] we proposed a totally blind detection method that is insensitive to noise uncertainty and performs robust detection using only few data samples, allowing short sensing time and relatively small complexity. Using the fact that the $\mathrm{CA}$ of the received signal is sparse (takes non zero values only at the cyclic frequencies), we exploited its property by applying a sparse approximation approach to this problem and use it for detection. This sparse approximation approach of the CA applied to spectrum sensing was firstly introduced in [9]. This approach, can be observed as an application of the sparse representation techniques, a topic that has been detailed through many fields in signal processing [10]-[12], and whose theoretical aspects focus more recently on the so-called compressed-sensing [13], [14]. In [15], we showed that the sparse CA function can be found using sparse representation technique that requires fewer samples than the classical estimator used in [6], while maintaining the same Mean Square Error (MSE) when compared to the theoretical curve of the CA. The idea of the proposed test in [1] is to take two small consecutive slots, and to compare their CA after applying the sparse representation technique. This algorithm detects the presence of signal when the two obtained CA are very close. We also showed in [1] that for short observation time under the same SNR the blind detector 
proposed in [1], outperformed the non blind detector proposed in [6], we also concluded that in order to achieve the same detection performance less samples (about 10 times less) were needed than the detector used in [6] but with relatively more complexity.

In this paper we propose a new totally blind algorithm, this new blind detection algorithm not only exploits the sparse property of the CA, but also exploits the property of its "symmetry" in order to enhance the detection performance using the same total number of samples and only one slot rather than two to detect.

The remaining part of this paper is organized as follows. In Section II, we present the system model adopted throughout this work. We introduce the background of the CA and how it could be estimated in Section III. A sparse model validation of the CA will be achieved in section IV. The new detection algorithm will be detailed in Section V. Section VI presents simulation results and discussions. Finally, Section VII presents the conclusions of this study and makes some suggestions for future work.

\section{SYSTEM MODEL}

The spectrum sensing detection problem consists of collecting a total number $N$ of samples $y(0), y(1), \ldots, y(N-1)$ from a given frequency band $B$. Denote $\mathbf{y}$ by the vector formed by $N$ samples, $\mathbf{y}=[y(0), . ., y(N-1)]^{t}$. $H_{1}$ and $H_{0}$ denote the binary hypotheses that a primary user is present and absent, respectively.

The binary hypotheses $\left(H_{0}, H_{1}\right)$ are defined in a way such that, under hypothesis $H_{1}$, and $k \in[0, . ., N-1]$, the $k^{t h}$ collected sample, $y(k)$, is composed of a primary user signal sample $x(k)$ plus an additive Gaussian noise sample $n(k) \sim$ $\mathcal{N}\left(0, \sigma_{n}^{2}\right)$, where $\mathcal{N}\left(m, \sigma^{2}\right)$ denotes the normal distribution with mean $m$ and variance $\sigma^{2}$. Under hypothesis $H_{0}$, the $k^{\text {th }}$ sample, $y(k)$, consists of the additive Gaussian noise sample $n(k)$. Hence, we can write:

$$
\left\{\begin{array}{l}
H_{0}: y(k)=n(k) \\
H_{1}: y(k)=x(k)+n(k)
\end{array}\right.
$$

Hence, the performance of any spectrum sensing method is indicated by two probabilities: the detection probability, $P_{d}$, and the probability of false alarm, $P_{f a}$.

\section{BACKGROUND}

In this section we will see how we can estimate the CA: $\mathbf{R}_{y y}^{\left(\tau_{0}\right)}(\alpha)=\mathbf{R}_{y y}(\alpha, \tau) \mid \tau=\tau_{0}$ of size $(N, 1)$ of the received signal in a classical method and then we will introduce a new way that allows us to estimate $\mathbf{R}_{y y}^{\left(\tau_{0}\right)}(\alpha)$ by using only $n$ samples with $n<N$ by exploiting the sparse property of $\mathbf{R}_{y y}^{\left(\tau_{0}\right)}(\alpha)$.

\section{A. A Classical Approach of Estimating the Cyclic Autocorre- lation}

In practice, telecommunication signals have special statistical characteristics. For example, digital modulated signals have non-random components, (such as bilateral spectrum due to sinusoidal carrier, the period symbol and the guard interval between orthogonal frequency division multiplexing (OFDM) symbols...). This particular property is called cyclostationarity which means that the statistical parameters of the signal vary periodically in time.

1) Cyclic Autocorrelation: A discrete time zero-mean (almost) cyclostationary process, $y(t)$, is characterized by the property that its time-varying autocorrelation $r_{y y}(t, \tau)=$ $E\{y(t) y(t+\tau)\}$ is periodic in time with a period called the cyclic period $T_{0}$, it has then a Fourier Series (FS) with respect to time $t$, given as [6]:

$$
r_{y y}(t, \tau)=\sum_{\alpha \in \mathcal{A}_{\alpha}} R_{y y}(\alpha, \tau) e^{j 2 \pi \alpha t}
$$

where the sum is performed over multiple integer of the fundamental cyclic frequency $\alpha_{f}=\frac{1}{T_{0}}$ of the received signal. $\mathcal{A}_{\alpha}=\left\{\alpha=\frac{k}{T_{0}}, k \in \mathbb{Z}\right\}$ is the set of cyclic frequencies and the Fourier coefficient $R_{y y}(\alpha, \tau)$, called the cyclic autocorrelation function, is given by

$$
R_{y y}(\alpha, \tau)=\lim _{T \rightarrow \infty} \frac{1}{T} \sum_{t=0}^{T-1} r_{y y}(t, \tau) e^{-j 2 \pi \alpha t}
$$

this function is continuous over the variable $\tau$ and takes non zero values for few $\alpha \neq 0$. We note that when the values of $y(t)$ are real (our case), $R(\alpha, \tau)$ presents the following symmetry properties [16]:

$$
\begin{aligned}
& R_{y y}(\alpha, \tau)=R_{y y}^{*}(-\alpha, \tau) \\
& R_{y y}(\alpha, \tau)=R_{y y}(\alpha,-\tau)
\end{aligned}
$$

With $R_{y y}^{*}(-\alpha, \tau)$ denotes the complex conjugate of $R_{y y}(-\alpha, \tau)$. Finally for $\alpha=0, R_{y y}(\alpha, \tau)$ becomes the classical autocorrelation function.

It is shown in [16] that the expression of the CA $R(\alpha, \tau)$ of a linearly modulated signal, with $T_{s}$ the symbol period, and $\tau$ a given delay, is null except when $\alpha$ takes an integer multiple of $\frac{1}{T_{s}}$ :

$R_{y y}(\alpha, \tau)=\left\{\begin{array}{l}\frac{1}{T_{s}} e^{-j 2 \pi \alpha \epsilon} \int_{-\infty}^{\infty} g\left(t-\frac{\tau}{2}\right) g^{*}\left(t+\frac{\tau}{2}\right) e^{-j 2 \pi \alpha t} d t \\ 0 \text { for } \alpha \neq \frac{k}{T_{s}}, k \in \mathbb{Z}^{*}\end{array}\right.$

with $\epsilon$ a random or unknown delay, $g(t)$ the temporal impulse response of the emission filter. From the previous equation one can derive the theoretical expression of the norm of the CA when using a rectangular window $g(t)$ defined in the temporal domain as:

$$
g(t)= \begin{cases}1 & |t| \leq \frac{T_{s}}{2} \\ 0 & \text { elsewhere }\end{cases}
$$

then, the obtained result is:

$$
\left\|R_{y y}(\alpha, \tau)\right\|_{2}=\left\{\begin{array}{l}
\frac{\left(T_{s}-\tau\right)}{T_{s}} \operatorname{sinc}\left(\alpha\left(T_{s}-\tau\right)\right) \\
0 \text { for } \alpha \neq \frac{k}{T_{s}}, k \in \mathbb{Z}^{*}
\end{array}\right.
$$


2) Cyclic Autocorrelation Estimation: In order to estimate the CA over $(\alpha, \tau)$ where $\alpha$ is the cyclic frequency and $\tau$ is a constant delay, we use the unbiased estimator used in [6]:

$$
\hat{R}_{y y}^{(N)}(\alpha, \tau) \cong \frac{1}{N} \sum_{t=0}^{N-1} y(t) y(t+\tau) e^{-j 2 \pi \alpha t}
$$

we define

$$
f_{\tau}(t)=y(t) y(t+\tau)
$$

then (6) can be written as:

$$
\hat{R}_{y y}^{(N)}(\alpha, \tau) \cong \frac{1}{N} \sum_{t=0}^{N-1} f_{\tau}(t) e^{-j 2 \pi \alpha t}
$$

we define the vector $\mathbf{f}_{\tau}$ by:

$$
\mathbf{f}_{\tau}=\left[f_{\tau}(0), f_{\tau}(1), \ldots, f_{\tau}(N-1)\right]^{t}
$$

and the vector $\hat{\mathbf{R}}_{y y}^{\left(\tau_{0}\right)}(\alpha)$ of $N$ elements by:

$$
\begin{aligned}
\hat{\mathbf{R}}_{y y}^{\left(\tau_{0}\right)}(\alpha)= & {\left[\hat{R}_{y y}^{(N)}\left(-\alpha_{\max }, \tau_{0}\right), \hat{R}_{y y}^{(N)}\left(-\alpha_{\max }+\delta_{\alpha}, \tau_{0}\right), \ldots\right.} \\
& \left., \hat{R}_{y y}^{(N)}\left(\alpha_{\max }, \tau_{0}\right)\right]^{t}
\end{aligned}
$$

where $\delta \alpha=\frac{2 \alpha_{\max }}{N}$ represents the resolution step. After observing at the expression (7), one can observe that the CA vector $\hat{\mathbf{R}}_{y y}^{\left(\tau_{0}\right)}(\alpha)$ of the received signal in the domain $\left[-\alpha_{\max }, \alpha_{\max }\right]$ is nothing but a scaled version of the Discreet Fourier Transform (DFT) of $\mathbf{f}_{\tau_{0}}$ :

$$
\hat{\mathbf{R}}_{y y}^{\left(\tau_{0}\right)}(\alpha)=\frac{1}{N} \operatorname{DFT}\left(\mathbf{f}_{\tau_{0}}\right)
$$

this last observation will be used in the next subsection to justify the choice of the dictionary, that is going to be used in the sparse representation, as it is going to be explained.

\section{B. Exploiting Sparsity}

In this paper we propose to exploit the sparse property of the CA, $\mathbf{R}_{y y}^{\left(\tau_{0}\right)}(\alpha)$. We can then reconstruct the vector $\hat{\mathbf{R}}_{y y}^{\left(\tau_{0}\right)}(\alpha)$ over $\alpha \in\left[-\alpha_{\max }, \alpha_{\max }\right]$ of length $N$ and resolution step $\delta \alpha=\frac{2 \alpha_{\max }}{N}$; by using only $n$ data samples (or observations) instead of using $N$ samples with $n<N$.

We propose then to apply a sparse representation approach based on representing the $n$ first elements of $\mathbf{f}_{\tau_{0}}$ over a redundant complex basis of $N$ cisoids at equispaced frequencies of spacing step $\delta_{\alpha}$, or equivalently on the columns vectors (also known as atoms) of the $(n, N)$-dimensional matrix denoted $A$ (the dictionary) which is the sub-matrix, formed by the $n$ first rows of $\bar{F}$, the conjugate of the $N$-dimensional square Fourier matrix $F$ (since $\hat{\mathbf{R}}_{y y}^{\left(\tau_{0}\right)}(\alpha)$ is nothing but a scaled version of the DFT of $\mathbf{f}_{\tau_{0}}, A$ is constructed using the rows of $\bar{F}$ ).

We recall that the Fourier matrix $F$ of order $N$ has the element $(p, q)$ equal to $e^{-2 i \pi(p-1)(q-1) / N}$. This matrix satisfies many properties such as the symmetry. We note also that $F \bar{F}=N I_{N}$, with $I_{N}$ the identity matrix. Then the DFT of a $N$-dimensional signal $y$ is given by $Y=F y$ and the Inverse Discrete Fourier Transform (IDFT) of $Y$ is $y=(1 / N) \bar{F} Y$.
We define $\mathbf{b}^{\left(\tau_{0}\right)}$ as an $(n, 1)$ column vector composed of the first $n$ elements of $\mathbf{f}_{\tau_{0}}$. The problem consists of solving the system:

$$
A \mathbf{r}^{\left(\tau_{0}\right)}=\mathbf{b}^{\left(\tau_{0}\right)}
$$

Then the solution $\hat{\mathbf{r}}^{\left(\tau_{0}\right)}$ which is an $(N, 1)$ column vector will represent the estimated CA, $\hat{\mathbf{R}}_{y y}^{\tau_{0}}(\alpha)$ over $\left[-\alpha_{\text {max }}, \alpha_{\text {max }}\right]$. This is a linear inverse problem with sparseness constraint, which is NP-hard, because for $N>n$ there are infinite solutions $\mathbf{r}^{\left(\tau_{0}\right)}$ that satisfy $A \mathbf{r}^{\left(\tau_{0}\right)}=\mathbf{b}^{\left(\tau_{0}\right)}$. The sparse problem is given by:

$$
\hat{\mathbf{r}}^{\left(\tau_{0}\right)}=\min \left\|\mathbf{r}^{\left(\tau_{0}\right)}\right\|_{0}, \text { s.t. } \quad A \mathbf{r}^{\left(\tau_{0}\right)}=\mathbf{b}^{\left(\tau_{0}\right)}
$$

Where $\|\cdot\|_{0}$ is the $l_{0}$-norm, simply defined by the number of non-zero entries in a vector.

An easier and intuitive approach, is to transforme the sparseness constraint over $\mathbf{r}^{\left(\tau_{0}\right)}$ into a convex optimization problem that gives a good and near optimal solutions [17]. Many efficient algorithms exist to find approximative solution for this kind of problems, from these algorithms we mention the Basis Pursuit (BP), the iterative algorithm called the Matching Pursuit (MP), the Orthogonal Matching Pursuit (OMP) [18] which is based on a variation of the earlier algorithm MP, LASSO etc. However, In our proposed algorithm, we used the OMP, which is going to be detailed in the next section, due to its simplicity and moderate complexity.

\section{Sparse Model VAlidation}

In this section we will recall the OMP algorithm that we are going to use in the new blind detection algorithm, then a model validation of the sparse reconstruction applied to a telecommunication signal will be done. Finally, we will recall the result presented in [15] that shows that the MSE of the obtained CA is lower when applying compressed sensing technique rather than classical method, using the same number of samples.

\section{A. The Orthogonal Matching Pursuit Algorithm}

Orthogonal Matching Pursuit (OMP) deliver an approximative solution to (8) by solving the problem (10) using an iterative process.

$$
\hat{\mathbf{r}}^{\left(\tau_{0}\right)}=\min \left\|\mathbf{r}^{\left(\tau_{0}\right)}\right\|_{0}, \quad \text { s.t. }\left\|A \hat{\mathbf{r}}^{\left(\tau_{0}\right)}-\mathbf{b}^{\left(\tau_{0}\right)}\right\|_{2} \leq \rho
$$

with $\rho$ is the maximal tolerated reconstruction error. At each iteration $l$ the locally optimum solution $\hat{\mathbf{r}}_{l}^{\left(\tau_{0}\right)}$ is calculated. This is done by finding at each iteration, the atom a in $A$ which is most correlated with the residual vector $\operatorname{res}_{l}$. The residual vector is initialized at the beginning to the vector that is required to be approximated i.e. $\mathbf{r e s}_{0}=\mathbf{b}^{\left(\tau_{0}\right)}$ then it is adjusted after each iteration taking into account the new approximation obtained using the new updated set of selected atoms. OMP uses a least-square minimization step at each iteration in order to improve the new obtained approximation and to update the residual vector as well. We note that in the OMP a new atom a is chosen after each iteration, this is to avoid a slow convergence to the solution. The detailed algorithm can be found in [18]. 


\section{B. Model Validation}

In order to validate our proposed method and to verify that the CA, $\hat{\mathbf{R}}_{y y}^{\left(\tau_{0}\right)}(\alpha)$ with $N$ elements can be reconstructed using only $n<N$ samples, for a given delay $\tau_{0}$ (in our example we choose $\tau_{0}=3 \cdot T_{e}$ with $T_{e}$ the sampling period) by applying compressed sensing technique, a BPSK modulation was used in order to simulate the transmitted signal, with $\alpha_{f}=\frac{1}{T_{s}}=$ 10000 the fundamental cyclic frequency where $T_{s}$ refers to the symbol period of the BPSK. Figure 1 illustrates, the CA of the BPSK modulation without filtering at the emission obtained using:

- the sparse approximation technique applying the OMP algorithm with a fixed number of iterations, employing a dictionary matrix $A$ of size $(n, N)$ as an input, with $n=1000$ and $N=4000$, in order to solve (8)

- the estimator (6) using 1000 samples

- the curve that represents the theoretical expression of the CA given in (5).

One can observe that when using the same number of samples (1000) to estimates the CA using the OMP, we obtain closer results compared to the theoretical curve than the curve obtained using (6). As a matter of fact, we can check on Figure 2 (a scaled version of Figure 1) the estimation noise on the curve that is obtained using (6), while no noise is observed for the curve that represents the compressed sensing technique result. Furthermore, one can observe a perfect estimation on the positions of the spikes, at integer multiple of the fundamental cyclic frequency when using both methods. This result validates our proposed sparse model of the CA for telecommunication signals.

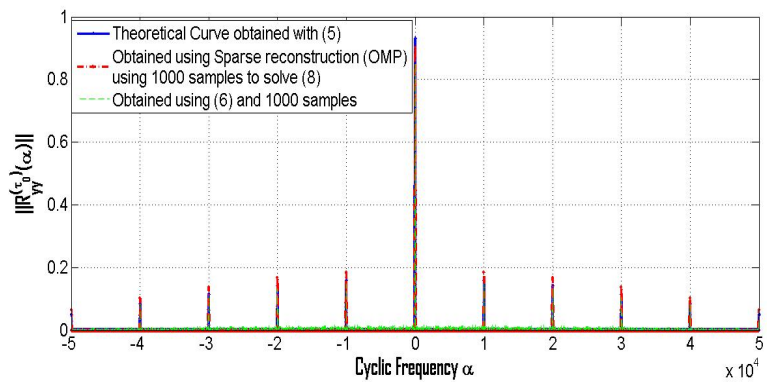

Fig. 1. The norm of the CA vector $\mathbf{R}_{y y}^{\left(\tau_{0}\right)}(\alpha)$, of a BPSK signal of fundamental cyclic frequency $\alpha_{f}=\frac{1}{T_{s}}$, with $\tau_{0}=3 . T_{e}$, obtained using (6), also compared to the theoretical curve (5) and to the norm of the CA obtained by solving (8) in order to find $\hat{\mathbf{r}}^{\left(\tau_{0}\right)}$ using the OMP. For both methods (the OMP and the estimator(6)), 1000 samples where used.

\section{Mean Square Error Comparison}

In Figure 3 we recall the result obtained in [15] that shows the $M S E$ between the theoretical curve and both: the curve obtained, using the classical method (formula (6)), and the one using compressed sensing via the OMP algorithm for different number of samples. One can conclude that using the compressed sensing offers better $M S E$ values than the classical method since it exploits the sparse property of the

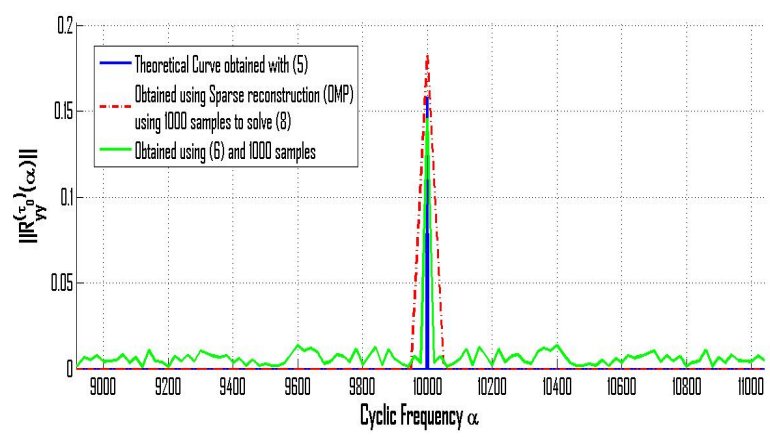

Fig. 2. Part of Figure 1 in scaled version in order to show clearly the estimation noise when using the classical estimator (6).

CA, especially when working with few samples we have the larger $M S E$ differences.

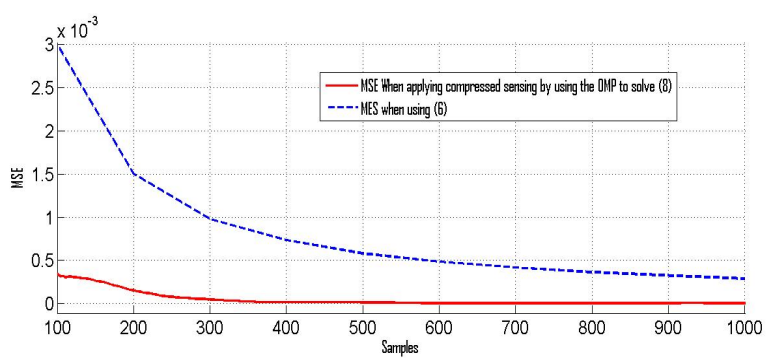

Fig. 3. The MSE (in linear representation) between the theoretical curve and respectively the CA obtained using the estimator (6), and the CA obtained by applying the compressed sensing technique to solve (8) using the OMP algorithm.

\section{The New Proposed Blind Detector}

In order to decide between $H_{0}$ and $H_{1}$, we propose in this paper a new blind method to detect, based on compressed sensing and on the symmetry property of the CA given in (3).

\section{A. Symmetry Property}

By taking the norm of both side of (3) one can obtain:

$$
\left\|R_{y y}(\alpha, \tau)\right\|_{2}=\left\|R_{y y}(-\alpha, \tau)\right\|_{2}
$$

We can also verify the property given in (11) by looking at Figure 1, we can see that the CA have a symmetry across the axe $\alpha=0$. The main idea of the proposed detector is to reconstruct partially the CA using few samples by applying compressed sensing and then to verify the symmetry of the obtained CA. If the obtained CA approximatively verifies (11) then $H_{1}$ is chosen otherwise $H_{0}$ is chosen. It is important to note that under $H_{0}$ the CA verify the property (11) as well, since $y(t)$ is real in both cases, but the probability to obtain a symmetrical CA under $H_{0}$, when using few number of iterations of the OMP algorithm to reconstruct partially the CA is very small as it is going to be explained in this section. 


\section{B. The New Proposed Algorithm}

Let $\mathbf{b}^{\left(\tau_{0}\right)}$ the vector as defined previously, we fix $l$ (odd) the number of iterations of the OMP algorithm in order to solve $\mathbf{b}^{\left(\tau_{0}\right)}=A \mathbf{r}^{\left(\tau_{0}\right)}$. Then the obtaining vector $\hat{\mathbf{r}}_{l}^{\left(\tau_{0}\right)}$ will be almost composed of zero elements, except for $l$ non zero elements (equal to the number of iterations of the OMP). We define by $I N D_{\text {sym }}^{\left(\tau_{0}\right)}$ the symmetry index of $\hat{\mathbf{r}}_{l}^{\left(\tau_{0}\right)}$. To calculate $I N D_{\text {sym }}^{\left(\tau_{0}\right)}$ we ignore the element of $\hat{\mathbf{r}}_{l}^{\left(\tau_{0}\right)}$ with the maximum amplitude that corresponds to $\alpha=0$, and then $I N D_{s y m}^{\left(\tau_{0}\right)}$ is obtained by calculating the mean value of the abscissa of the $l-1$ remaining elements. The more $I N D_{s y m}^{\left(\tau_{0}\right)}$ is close to zero the more ideal the symmetry is. An ideal symmetry is obtained when there is no noise and it is manifested by $I N D_{s y m}^{\left(\tau_{0}\right)}=0$. $I N D_{\text {sym }}^{\left(\tau_{0}\right)}$ is obtained using the following equation:

$$
I N D_{s y m}^{\left(\tau_{0}\right)}=\frac{1}{l-1} \sum_{j=2}^{l} \alpha_{j}
$$

Figure 4 illustrates a calculation example of $I N D_{\text {sym }}^{\left(\tau_{0}\right)}$ for a given $\tau_{0}$ and for a fixed $l=3$. We have in this ideal case $I N D_{\text {sym }}^{\left(\tau_{0}\right)}=\frac{1}{2}\left(\alpha_{2}+\alpha_{3}\right)=0$

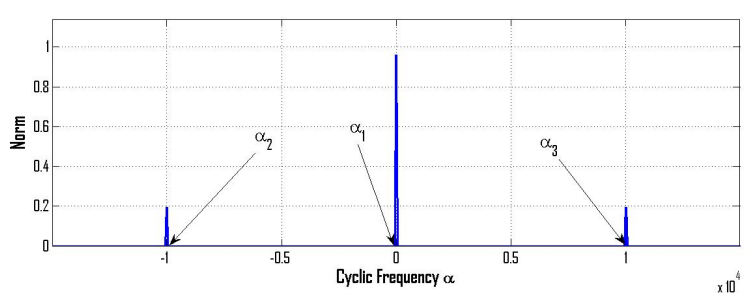

Fig. 4. Calculation example of $I N D_{s y m}^{\left(\tau_{0}\right)}$ for a given $\tau_{0}$ and for a fixed $l=3$. We have in this ideal case $I N D_{s y m}^{\left(\tau_{0}\right)}=\frac{1}{2}\left(\alpha_{2}+\alpha_{3}\right)=0$

Before taking the final decision we note that $\hat{\mathbf{r}}_{l}^{(\tau)}$ should be estimated for different values of $\tau, \tau \in\left\{\tau_{0}, \tau_{1}, \ldots, \tau_{M}\right\}$, because it is not necessary that the second order statistic $R(\alpha, \tau)$ takes non zero values for any value of $\tau$, even if $\alpha$ is a cyclic frequency of the received signal (cf. [6]). This means that the algorithm will calculate $M+1$ different values of $I N D_{s y m}^{\left(\tau_{i}\right)}$ (using $M+1$ times the OMP algorithm) and the final decision will be made using the equivalent index obtained by combining all the obtained indexes making a soft decision:

$$
I N D_{\text {sym }}^{(e q u)}=\frac{1}{M+1} \sum_{i=0}^{M}\left|I N D_{\text {sym }}^{\left(\tau_{i}\right)}\right|
$$

finally $I N D_{\text {sym }}^{(e q u)}$ will be compared to a positive threshold $\xi$ in order to decide between $H_{0}$, and $H_{1}\left(I N D_{\text {sym }}^{(e q u)}<\xi\right)$. We note that the larger $\xi$ is, the higher the false alarm and the detection probabilities are, and vice versa.

\section{The Choice of the Number of Iterations}

The purpose of the detection algorithm is not to make a full reconstruction of the CA, but to detect the existence of a signal. For this reason it is judicious to choose $l$ in order to maximize the detection performance and to minimize the complexity of the detection algorithm as well.

1) An Odd Number of Iterations: The number of iterations $l$ should be an odd number in order to evaluate properly $\left|I N D_{\text {sym }}^{\tau_{0}}\right|$, because as mentioned before the algorithm needs to check the symmetry over the axe $\alpha=0$. The element in $\hat{\mathbf{r}}_{l}^{\left(\tau_{0}\right)}$ with the highest amplitude corresponds to $\alpha=0$ and is obtained after the first iteration of the OMP (since it has the highest amplitude), the remaining $l-1$ nonzero element that represents the cyclic frequencies values should be pair by pair symmetric over $\alpha=0$ (for each spike with cyclic frequency $\alpha$ corresponds another spike at $-\alpha$ having the same norm). This is the reason that $l$ should be an odd number.

2) A Minimal Number of Iterations: Two major reasons make it judicious to choose the minimal possible value of $l$, i.e $l=3$. The first reason is related to the complexity of the OMP algorithm which depends on the number of iterations $l$, then choosing $l$ as small as possible will minimize the complexity as much as possible. We note that the complexity of this new algorithm is the same as the blind proposed algorithm of [1], given by $O\left((M+1) \cdot n_{t o t} \cdot N^{\prime} \cdot l\right)$ which is nothing but the complexity of the OMP that can be found in [19], multiplied by $M+1$, where $n_{t o t}$ is the total number of samples used to detect, and $N^{\prime}$ the number of columns of the used dictionary.

The second major reason is to minimize the false alarms. Although property (11) is verified under $H_{0}$ the proposed detector can still be used to distinguish between $H_{1}$ and $H_{0}$; because under $H_{1}$ when applying the OMP, symmetrical atoms to $\alpha=0$ and close to an integer multiple of the fundamental cyclic frequency will be selected consecutively after consecutive iterations since they have the same non zero norm value (by definition the OMP selects the most correlated atoms with the residual vector at each iteration). In contrary under $H_{0}$ the CA function is zero, since noise doesn't have cyclic frequencies except for $\alpha=0$ (takes the value of the classical autocorrelation). For this reason under $H_{0}$, atoms will not be selected in a specific order minimizing the probability of obtaining a symmetrical reconstructed vector $\hat{\mathbf{r}}_{l}^{\left(\tau_{0}\right)}$ for a small value of $l$. We note that under $H_{0}$ the more $l$ increases a more complete reconstructed vector $\hat{\mathbf{r}}_{l}^{\left(\tau_{0}\right)}$ is made, and the probability of verifying the symmetry property increases generating false alarms.

\section{Simulation Results and Discussion}

\section{A. Detection Performance}

In this part, a BPSK modulation was used to achieve the simulations. A smaller matrix $A$ of size $(160,512)$ was used in order to obtain lower computation complexity. $l$ the number of iterations was fixed to 3 for the reasons mentioned above. We compared our blind detector to the one proposed in [1].

Figure 5, shows the detection performance versus the $S N R$, for a fixed observed false alarm rate of $15 \%$, using a total number of 160 samples for both methods, to make a fair comparaison. Two different sets of lags $\tau$ were used in the simulation of both detection methods the first set is composed only from 2 values of $\tau(M=1)$, while the second is 
composed from 5 different values $(M=4)$. Fisrt we can conclude that for any set of lags $\tau$ the new proposed method outperforms the method proposed in [1]. We can also check that when the number of used lags for detection increases $(M)$ the detection performance gets better for both methods as it is expected. Finally, we can see for example that for $M=4$, the new blind method reaches detection probability of 0.9 , versus only $P_{d}=0.42$ for the method proposed in [1] at $S N R=-2 \mathrm{~dB}$ which means that in these conditions the detector of [1] misdetects more than $50 \%$ the presence of the signal (misdetection probability $P_{m}=1-P_{d}=0.58$ ) versus only 0.1 for the new detector for the same: $P_{f a}$, calculation complexity, set of lags $(M)$, and observation time.

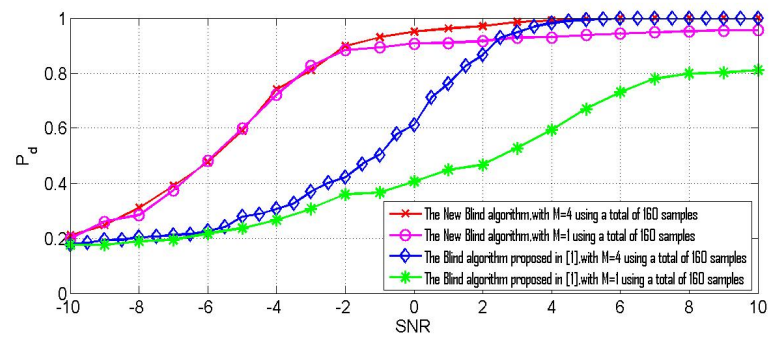

Fig. 5. Detection probability as function of the $S N R$ for a fixed false alarm $\left(P_{f a}=0.15\right)$ and for the same number of samples (160 samples), for both: the new proposed blind method and the one proposed in [1]. Two sets of lags $\tau$ where used ( $M=1$ and $M=4$ ) for both methods.

As mentioned in the previous section the purpose of the blind algorithm is to detect signal and not to make a full reconstruction for the $\mathrm{CA}$ function. In order to show that we obtain the best performance when the number of iterations $l$ is minimal, we simulated under $S N R=0 \mathrm{~dB}$, and for 160 samples, the false alarm probability as function of the iteration number $l$ taking the odd values from 1 to 21 . The simulation result is shown on Figure 6. The result validates that the lowest false alarm probability $\left(P_{f a}=0.04\right)$ is obtained for the minimal possible value of $l(l=3)$ and increases with it to reach $P_{f a}=0.64$ for $l=21$.

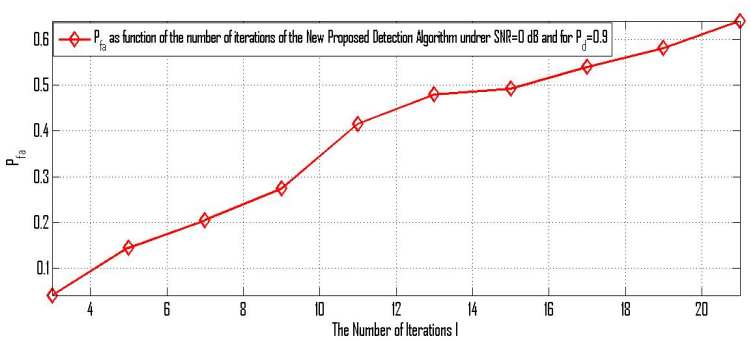

Fig. 6. False alarm probability as function of the iterations number $l$ (odd) given for a fixed detection probability $P_{d}=0.9$ and a fixed $S N R=0 \mathrm{~dB}$.

\section{CONCLUSION}

In this paper, based on our previous work of detecting the existence of primary users via compressed sensing blindly, we introduce a new blind detector by employing the use of the symmetry property of the CA to improve the detection performance. Simulation results of the reconstruction algorithm, detection probability and false alarm probability confirm the effectiveness and robustness of the proposed method. We also showed that we can achieve better detection by using minimal number of iterations when applying the OMP algorithm. Future work will include a theoretical study on the detection and false alarm probabilities. In addition, the sensitivity of the detection in the presence of interfering signals and the performance under fading channel will be studied.

\section{ACKNOWLEDGMENT}

Part of this work was supported by COST Action IC0902 "Cognitive Radio and Networking for Cooperative Coexistence of Heterogeneous Wireless Networks"

\section{REFERENCES}

[1] Z. Khalaf, A. Nafkha, and J. Palicot, Blind Spectrum Detector for Cognitive Radio using Compressed Sensing. IEEE GLOBECOM, December 2011, Houston, Texas, USA.

[2] L. LU, X. Zhou, U. Onunkwo and G. Y. Li, Ten years of research in spectrum sensing and sharing in cognitive radio, EURASIP Journal on Wireless Communications and Networking 2012,2012:28.

[3] S. M. Kay, Fundamentals of Statistical Signal Processing: Detection Theory, vol. 2, Prentice Hall, Upper Saddle River, NJ, USA, 1998.

[4] W. Jouini, Energy detection limits under log-normal approximated noise uncertainty, IEEE Signal Processing Letters, Vol. 18, NO.7, JULY 2011

[5] W.A. Gardner and C.M. Spooner, Signal interception: Performance advantages of cyclic feature detectors, IEEE Transaction on Communications, vol. 40, January 1992.

[6] A.V. Dandawate and G.B. Giannakis, Statistical tests for presence of cyslostationarity, IEEE Transactions on Information Theory, vol. 42, pp. 2355-2369, September 1994

[7] L. Lu, H. Wu, A Novel Robust Detection Algorithm for Spectrum Sensing, IEEE Journal on selected areas in communications, Vol. 29, No. 2, FEBRUARY 2011

[8] F. Socheleau, S. Houcke, P. Ciblat and A. Abdeldjalil Cognitive OFDM system detection using pilot tones second and third-order cyclostationarity, Signal processing, February 2011, vol. 91, n 2, pp. 252-268

[9] Z. Khalaf, A. Nafkha, and J. Palicot, Blind cyclostationary feature detector based on sparsity hypotheses for cognitive radio equipment. MWSCAS, 2011 the 54th IEEE International Midwest Symposium on Circuits and Systems, August 2011, Seoul,Korea.

[10] D.L. Donoho and X. Huo. Uncertainty principles and ideal atomic decomposition. IEEE Trans. on I.T., 47, 11, 2845-2862

[11] R. Gribonval and M. Nielsen. Sparse representations in unions of bases. IEEE Trans. on I.T. 49, 12, 3320-3325, Dec. 2003

[12] J.J. Fuchs. More on sparse representations in arbitrary bases. IEEE Trans. on I.T. 50, 6, 1341-1344, June 2004, also in 13th IFAC SYSID, 1357-1362, Rotterdam, 2003.

[13] E. Candes, J. Romberg and T. Tao, Robust uncertainty principles: Exact signal reconstruction from highly incomplete frequency information. IEEE T. on I.T., 52, 489-509, 2006.

[14] E. Candes and T. Tao, Near optimal signal recovery from random projections: Universal coding strategies? IEEE Trans. on I.T., 52, 54065425, 2006.

[15] Z. Khalaf, and J. Palicot, Exploiting Sparse Property of the Cyclic Autocorrelation Function for Cyclostationary Process 7th Karlsruhe Workshop on Software Radios, WSR'12, Karlsruhe, Germany, 7-8 March 2012

[16] William A.Gardner, Statistical Spectral Analysis: A Non-Probabilistic Theory,Prentice Hall, January 1988.

[17] J.J. Fuchs Identification of real sinusoids in noise, the Global Matched Filter approach. 14th Ifac-Ifors symposium on Identification and system Parameter Estimation, pp. 1127-1132, Saint-Malo, France, July, 2009

[18] G. Davis, S. Mallat, and M. Avellaneda, Greedy adaptive approximation, J. Constr. Approx. 13, pp. 57- 98, 1997.

[19] A. Doostan and H. Owhadi, A non-adapted sparse approximation of PDEs with stochastic inputs Journal of Computational Physics Volume 230, Issue 8, 20 April 2011, Pages 3015-3034. 